\title{
Network Health Improvement Techniques
}

\author{
Sinduja M S, KR.Rekha
}

\begin{abstract}
With the advancement in technology, there are recent developments in the micro-sensor devices that have accelerated the progress in the field of sensor networks. Sensing Point Areas (SPAs) uses are in various applications like battlefield monitoring detection of Enemy vehicles and environment surveillance. In all the application energy consumption is one of the constraints and is critical that affects the Network Health (NH) of the Sensor Area. Energy utilization in an efficient manner is crucially important to maintain a network in its operational condition for the longest time. Therefore, Network Health (NH) improvement techniques have gained more attention to extend the unmarked operation of battery-constrained WSNs. In this paper, we review the application of Wireless Sensor Networks (WSNs), the summary of various kinds of networks, energy consumption model, NH definition, and its improvement techniques.
\end{abstract}

Keywords: Wireless Sensor Network, Sensing Point Network, Internet of Things

\section{INTRODUCTION}

SENSING Point Network (SPN) history began from early 1980s with the usage of wireless voice Sensor Area [1]. For SPN one of the main limitations is the battery capacity of the SPs. The usage of the SPN over the years has been depicted in the Figure 1. As shown in Figure 1 the SPN are used for wide variety of applications [2]. The SPNs are used for very wide range of applications. SP is an entity in the Sensing Point Network (SPN) which can detect the data and is governed by chargeable value, storage volume and sensing tip. SPN will be classified into Multiple Group SPN and Single Group SPN. Figure 1 shows the applications of SPNs. Various applications make use of SPN namely Military Application, Environment Monitoring, Logistics, Transport Devices and Applications and Hospital Smart Health Clinics.Non-Group SPN will have the SPs spread in a parameterized end points of $x^{*} y$. Each SP can communicate to other SP using algorithms without any centralized SP. The example of Non-Group SPN within an area of $100 * 100$ is

Revised Manuscript Received on August 25, 2020.

* Correspondence Author

Sinduja M S*, Research Scholar, Department of ECE, SJBIT Research Centre, Bangalore, India. E-mail: sindujak29@gmail.com

Dr. K R. Rekha, Professor, Department of ECE, SJBIT, Bangalore, India. E-mail: rekha.sjbit@gmail.com

(C) The Authors. Published by Blue Eyes Intelligence Engineering and Sciences Publication (BEIESP). This is an open access article under the CC BY-NC-ND license (http://creativecommons.org/licenses/by-nc-nd/4.0/)

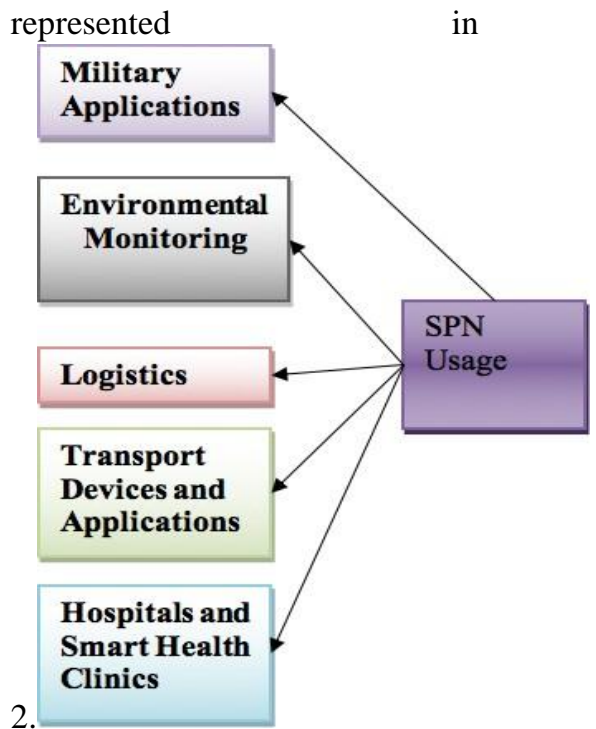

Figure

Fig. 1. Applications of SPNs

Group based SPN will have the SPs in multiple independent groups. Each group will have set of SPs. Each SP group will have Group Identifier SP which can send control packet between groups. Group based SPN can be divided into Equal SPN and Un- Equal SPN. For the case of Equal SPN all the groups will have N SPs. For the case of Un-Equal SPN the group can have its own number of SPs.

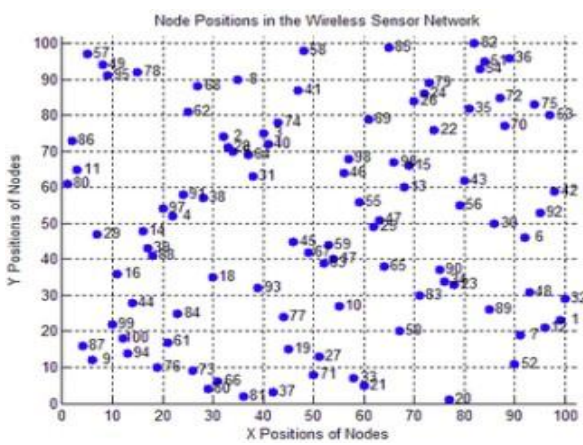

Fig. 2. Non - Group Sensor Area

Figure 3 shows 4 clusters and each of the clusters has a set of 10 SPs in the Sensor Area.

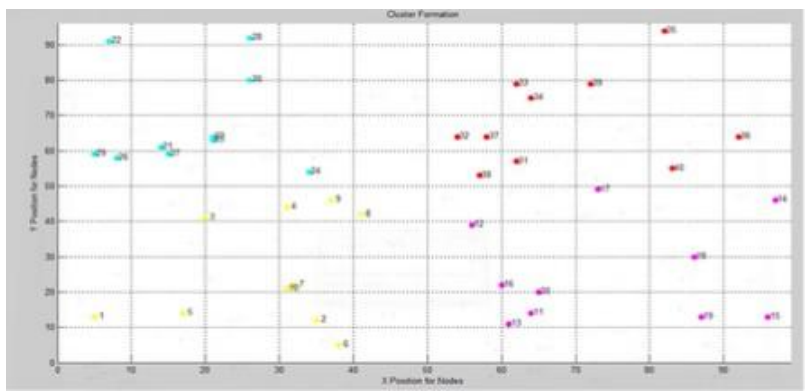

Fig. 3. Group Based Network

Blue Eyes Intelligence Engineering 


\section{Network Health Improvement Techniques}

The Cluster based Sensor Areas can be further divided into 2 sub Sensor Areas,

1) Homogenous Sensor Area

2) Non-Homogenous Sensor Area

Homogenous Sensor Area is the Sensor Area which has similar set of SPs in each of the clusters. Non-Homogenous Sensor Area is the Sensor Area which has different set of SPs in each of the clusters.

\section{ENERGY CONSUMPTION MODEL}

This section discusses the energy consumption model in SPN. When the distance between Node A and Node B is 'dis' and then certain communication data (Bn bytes) is sent [3]. The SP has majorly main components like transmitter component and amplifier component as shown in Figure 4 [4].

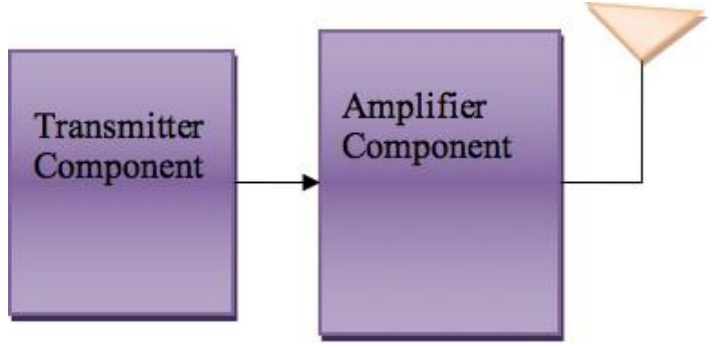

Fig. 4. Major Energy Dissipation Components

The amount of data transmission energy level can be defined as below

$E_{\text {datatx }}=B_{n} \cdot\left(E_{\text {txlevel }}+E_{\text {amp }} \cdot\right.$ dis $\left.^{2}\right)$

$B_{n}=$ bytes of data

Dis $=$ distance between SPs

$E_{\text {txlevel }}=$ data transmission energy

$E_{\text {amp }}=$ amplification factor

The destination SP will spend the following value of energy

$E_{\text {rxvalue }}=B_{n} \cdot E_{\text {txlevel }}$

The total energy is the summation of transmission level of energy and reception of energy.

$E_{C}=E_{\text {datatx }}+E_{\text {rxvalue }}$

$E_{C}=$ Bn Etxlevel $+B_{n} E_{a m p} \cdot$ dis $^{n}+B_{n}$ Etxlevel

The classical energy consumption model makes use of standard values as given in Table I [4].

Table- I: Energy Consumption Values

\begin{tabular}{|c|c|}
\hline Mode Level & Energy Value \\
\hline $\begin{array}{l}\text { Exlevel } \\
\text { Exxlevel }\end{array}$ & $50 \mathrm{~nJ} / \mathrm{bit}$ \\
\hline$E_{\text {amp }}$ & $100 \mathrm{pJ} / \mathrm{bit} / \mathrm{m}^{2}$ \\
\hline
\end{tabular}

When packets are sent the SP will lose its battery by a certain amount. The end path found using a routing method can be defined as below

$5-->8--->10---->12$
The transmission happens between links from SP5 to SP8, then from SP8 to SP10 and finally from SP10 to SP12.

Substituting the values from Table 1 in Equation 4. The energy consumed between SP5 to SP8 can be calculated as (Assuming $B_{n}=10$ )

$$
E_{C(S P 5, S P 8)}=10\left(2 * 50+100 * 30^{2}\right)
$$

Similarly, the energy consumed between SP8 to SP10 is the following

$$
E_{C(S P 8, S P 10)}=10\left(2 * 50+100 * 20^{2}\right)
$$

There is a direct relation between the coverage range of SPs and total energy consumed and is depicted in Figure 5 . The equal interval of distance 10 is maintained and then increased till a maximum limit of $100 \mathrm{~m}$. The direct proportionality can be seen as an incrementing factor of energy with distance.

\section{NETWORK HEALTH COMPUTATION}

The Network Health (NH) helps in maintaining the control and data packet flows in the network and it has multiple definitions [5]. Network Health can be defined as

$$
\text { NH = Count Healthy/CountNon Healthy }
$$

Where,

CountHealthy = Set SP who's Residual Energy (RE)

$>=$ Initial Battery (IB)/ 4

CountNonHealthy = Set SP who's Residual Energy $(\mathrm{RE})<$ Initial Battery (IB)/4

Initial Battery $\left(\mathrm{IB}_{1}\right)$ is the Initial Energy of an SP. If the value of $\mathrm{IB}_{1}$ is reduced by four times and is the current energy level of SP then such a SP is called as Non-Healthy SP [6].

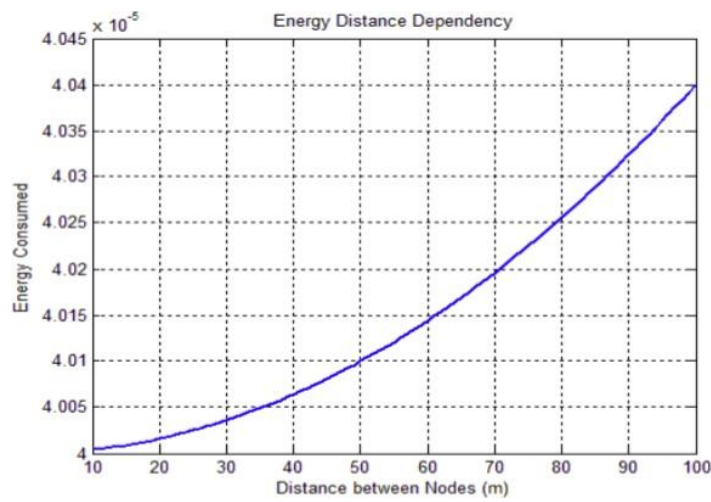

Fig. 5. Range v/s Energy Dissipation

The usage of a given SP multiple times on the routing path will cause more severe energy reduction and can be computed as follows,

Where,

$$
N E_{S P}=C E_{S P}-E C_{\text {link }}
$$

$\mathrm{CE}_{\mathrm{SP}}=$ Current Energy of SP

$\mathrm{EC}_{\text {link }}=$ Energy Consumption between links

When the network is first formed, each SP will have same amount of energy. Energy is consumed by the SP when it participates in the route repeatedly, a point will be reached when new energy value of the SP is less than 4 times the initial energy and hence SP becomes Non-Healthy.

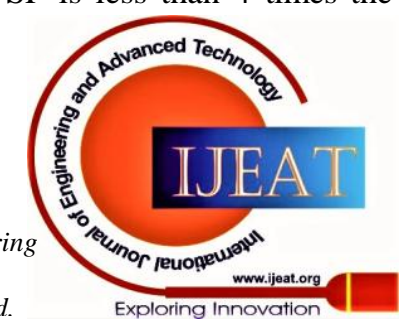




\section{NETWORK HEALTH IMPROVEMENT TECHNIQUES}

This section describes the various methods which can be used to improve the health ratio of the network.

\section{A. Energy Reaping}

The concept of getting the energy for the SPs through multiple means like thermal, solar and kinetic systems is referred as energy reaping.

Many real-world apps make use of Internet of Things (IOT). The concepts of reducing the consumption levels and then renewing the energy levels can improve the health of the network [6].

The data transmitted from area A to a different area B can be improved with the help of ER-SPN [7] which contains sensing points (SPs) for each home. The conventional techniques are improved by making modifications on the duty cycle, amount of power used for transmission and then data rate. Energy Reaping (ER) can help to recharge the SPs by making use of solar power [8]. When a SP is used repeatedly then its battery level becomes below certain threshold and hence effects the health of the network. The SP energy can be reworked with the help of ER [9].

\section{B. Transmission And Connection Concept}

Food security is important concept for any country and hence monitoring of crop yields through SPs help is achieving this goal [10]. The health of the network can be improved by providing better transmission and then good link with respect to base station [11].

The transmission within the area can be done with the help of healthy SPs for a specific period of time T. The data is sent towards the control center SP with the help of multiple SPs [12]. The SPs can take either ON mode or OFF mode to scan the region so that health can be maximized [13]

The problem of transmission can be resolved by doing a logical mapping of an area. Classification of area based on number of SPs and amount of data transfer can be used to maximize network health [14].

\section{Access To Opportunity Based Transmission And Scheduler}

The disturbance in the signal with respect to multiple parameters namely time, position of SPs and then frequency is called as fading.

When the data has to be sent from one SP to the destination $\mathrm{SP}$, a route trace is created which is a list of SPs participating in the trace. The health of the network can be improved by placing relay SPs within the area. With the help of CSD and RE, network health can be improved. The SPs used in data transfer can adjust the power based on RE and CSD [15]. Before transmission of data, SP checks the quality parameter and if the value is higher than threshold then only data is sent so that network health can be improved [16]. If the data received by the destination occurs for every time duration ' $T$ ' then SPs can be made to undergo OFF mode and then switch to ON mode during packet arrival [17]. Two kinds of such scheduler can be used either fixed or joint scheduling. The joint scheduling reduces the amount of Energy Dissipation (ED) and hence improves network health [18].

\section{Beam Steering}

The highest signal strength generation towards the actual SP and then side lobes towards the non-target SPs is called as Beam Steering [19] - [20].

Multiple concepts like signal multiple with itself, signal multiple with different signal, computation of weights using Sample Matrix Inverse Least Mean Square are used to achieve beam steering. The energy wastage can be shared among the SPs and maximum transmission of data can be achieved with the help of set of Sensors on each SP rather than single sensor. This can also help in reduction of load and then replication of data can be done while battery is critical [21]. while doing beam steering, energy levels of the SPs can be taken into consideration to achieve significant improvement as compared to conventional techniques. Since the energy consumed at SP is smaller than energy received by SP the minimum sample rate is maximized [22]. Beacons can be used to transfer the power to the SPs to maximize the total average received power [23].

\section{E. Allocation Of Assets}

The routing, schedule of data, location of SPs, maximum throughput and rate adaptation are most important tasks, which are responsible for complete maintenance of network health. Energy Efficient paths can be achieved by keeping SPs in ON and OFF mode with respect to MAC layer [24]. A network layer contains multiple flavors like link, route and MAC. Power allocation, scheduling of data across links and link energy consumption can be optimized across these three flavors in order to reduce the ED which will improve network health [25]. The quality of water in the rivers can be detected with the help of SPs by making use of ON and OFF mode [26].

\section{F. Data Acquisition}

Route trace will contain set of SPs. If any of the SP fail, then data will contain errors and also dropping of packets can happen. By making use of Low Rank Parity Check (LRPC), errors can be corrected and then decoding rate can be efficient [27].

The data is sent to the base station by making use of SPs. When the flow of data is high then network health will reduce. Multiple parameters namely limited memory, reduced energy and complexity of computation expose the weakness of SPs group [28].

The decrease of Energy Consumption (EC) will be based on combination of zone and tree-based method that will help in increasing the energy efficiency. This will increase the network health [29]. This method will make use of hierarchical techniques in order to increase the network health.

\section{G. Energy Consumption Reduction}

Different applications usage like transmission of medical data, environment related data, transportation plans make use of SPN. The process of re-transmission in order to reduce redundancy and increase the reliability. Since the algorithms does not take energy consumption factor into consideration, it can be reduced by making use of multi-path routing [30]. 


\section{Network Health Improvement Techniques}

When multiple SP are combined, they form a region. The SP of one region will send data to SP head of that region which in turn takes the responsibility of sending data towards the destination base station [3]. Multiple methods can be used in the selection of SP head.

Centrality Approach will be used to elect the SP head. whichever is closer to center is computed. When a SP is used repeatedly along multiple paths it will eventually become unhealthy. SPA method on top of LEACH is established to achieve better balance of data and lesser number of loops.

The optimization of SPN will make use of energy modelling along with energy consumption value computation. The model will consider MAC and physical layer for the combination of Additive white Gaussian noise (AWGN) and Non-AWGN concept [4].

The value of mean for energy, ratio of drop packets, latency is used for both Minimum shift keying (MSK) and quadrature phase shift keying (OQPSK16) [31]. SPs will have multiple communication range, conditioning of data and then sensing capability.

The processing capacity can be improved by using collaboration between layers which can be used for task mapping along with scheduling of data with reduction of energy [32]. The minimization of energy value along with physical layer computation will be used based on Rayleigh fading data [5].

Optimization of transmission power will be based on minimum energy consumption [6] and then data is sent towards the base station. SPN has limited energy, data storage capability and battery energy and will limit the network health. The SPN will group SP and then send data from normal SP to SP head then SP head will send data to base station. The threshold for data reduction will help in pre-processing along with minimization of energy consumption. Redundancy is removed at each SP so that more transmission can be achieved [7].

\section{CONCLUSION}

The work presents the category of hierarchical SPN and Nonhierarchical SPN. A comprehensive study on the energy consumption model describes the reduction in the energy of nodes that happens on the routing path. Various network health improvement techniques like Energy Reaping, transmission and connection concept, access to opportunity based transmission and scheduler, bean steering, allocation of assets, data acquisition, and energy consumption reduction is presented in the literature.

\section{REFRENCES}

1. A. Sharif, V. Potdar, and E. Chang, "Wireless multimedia sensor network technology: A survey," 07 2009, pp. 606-613.

2. K. Romer and F. Mattern, "The design space of wire-less sensor networks," IEEE Wireless Communications, vol. 11, no. 6, pp. 54-61, 2004.

3. M. Abo-Zahhad, M. Farrag, and A. Ali, "Modeling and optimization of energy consumption in wireless sensor networks," in 2015 Tenth International Conference on Computer Engineering Systems (ICCES), 2015, pp. 295- 300.

4. W. R. Heinzelman, A. Chandrakasan, and H. Balakr-ishnan, "Energy-efficient communication protocol for wireless microsensor networks," in Proceedings of the 33rd Annual Hawaii International Conference on System Sciences, 2000, pp. 10 pp. vol.2-.

5. L. R. V. and E. Baburaj, "Efficient approach to maximise WSN lifetime using weighted optimum storage-node placement, efficient and energetic wireless recharging, efficient rule-based node rotation and critical-state-data-passing methods," IET Networks, vol. 6, no. 6 , pp. 203-217, 2017. [Online]. Available: https: //doi.org/10.1049/iet-net.2017.0035

6. S. Perez, J. A. C. Fuertes, and M. Coupechoux, "Odmac++: An iot communication manager based on energy harvesting prediction," in 2017 IEEE 28th An-nual International Symposium on Personal, Indoor, and Mobile Radio Communications (PIMRC), 2017, pp. 1-7.

7. E. Simoen and C. Claeys, "On the impact of the capture rates on the generation/recombination lifetime ratio of a single deep level," IEEE Transactions on Electron Devices, vol. 46, no. 7, pp. 1487-1488, 1999.

8. P. K. Sharma, Y.-S. Jeong, and J. H. Park, "Eh-hl: Effective communication model by integrated eh-wsn and hybrid lifi/wifi for iot," IEEE Internet of Things Journal, vol. 5, pp. 1719-1726, 2018.

9. G. Jackson, S. Ciocoiu, and J. A. McCann, "Solar energy harvesting optimization for wireless sensor networks," in 2017 IEEE Global Communications Conference, GLOBECOM 2017, Singapore, December 4-8, 2017. IEEE, 2017, pp. 1-6. [Online]. Available: https://doi.org/10.1109/GLOCOM.2017.8254851

10. X. Deng, B. Wang, W. Liu, and L. T. Yang, "Sensor scheduling for multi-modal confident information cover-age in sensor networks," IEEE Transactions on Parallel and Distributed Systems, vol. 26, no. 3, pp. 902-913, 2015.

11. C.-P. Chen, S. Mukhopadhyay, C.-L. Chuang, M.-Y. Liu, and J.-A. Jiang, "Efficient coverage and connectivity preservation with load balance for wireless sensor net-works," Sensors Journal, IEEE, vol. 15, pp. 48-62, 012015.

12. Q. Zhao and M. Gurusamy, "Lifetime maximization for connected target coverage in wireless sensor networks," IEEE/ACM Transactions on Networking, vol. 16, no. 6, pp. 1378-1391, 2008.

13. Z. Lu, W. W. Li, and M. Pan, "Maximum lifetime scheduling for target coverage and data collection in wireless sensor networks," IEEE Transactions on Vehic-ular Technology, vol. 64, no. 2, pp. 714-727, 2015.

14. T. O. Olasupo and C. E. Otero, "A framework for optimizing the deployment of wireless sensor networks," IEEE Transactions on Network and Service Management, vol. 15, no. 3, pp. 1105-1118, 2018.

15. J. Matamoros and C. Anton-Haro, "Opportunistic power allocation and sensor selection schemes for wireless sensor networks," IEEE Transactions on Wireless Com-munications, vol. 9, no. 2, pp. 534-539, 2010.

16. C. V. Phan, Y. Park, H. H. Choi, J. Cho, and J. G. Kim, "An energy-efficient transmission strategy for wireless sensor networks," IEEE Trans. on Consum. Electron., vol. 56, no. 2, p. 597-605, May $2010 . \quad$ [Online]. Available: https://doi.org/10.1109/TCE.2010.5505976

17. J. Kim, X. Lin, N. B. Shroff, and P. Sinha, "Minimizing delay and maximizing lifetime for wireless sensor net-works with anycast," IEEE/ACM Transactions on Net-working, vol. 18, no. 2, pp. 515-528, 2010.

18. F. Liu, C. Tsui, and Y. J. Zhang, "Joint routing and sleep scheduling for lifetime maximization of wireless sensor networks," IEEE Transactions on Wireless Communica-tions, vol. 9, no. 7, pp. 2258-2267, 2010.

19. F. Alavi, K. Cumanan, Z. Ding, and A. G. Burr, "Beam-forming techniques for nonorthogonal multiple access in $5 \mathrm{~g}$ cellular networks," IEEE Transactions on Vehicular Technology, vol. 67, no. 10, pp 9474-9487, 2018.

20. A. Senapati, K. Ghatak, and J. S. Roy, "A compara-tive study of adaptive beamforming techniques in smart antenna using lms algorithm and its variants," in 2015 International Conference on Computational Intelligence and Networks, 2015, pp. 58-62.

21. Z. Han and H. V. Poor, "Lifetime improvement in wire-less sensor networks via collaborative beamforming and cooperative transmission," IET Microwaves, Antennas Propagation, vol. 1, no. 6, pp. 1103-1110, 2007.

22. R. Du, A. Ozc, elikkale, C. Fischione, and M. Xiao, "Towards immortal wireless sensor networks by optimal energy beamforming and data routing," IEEE Transac-tions on Wireless Communications, vol. 17, no. 8, pp. 5338-5352, 2018.

23. Z. Lu, W. Li, and M. Pan, "Maximum lifetime scheduling for target coverage and data collection in wireless sensor networks," IEEE Transactions on Vehicular Technology, vol. 64, pp. 714-727, 022015

Published By:

Blue Eyes Intelligence Engineering and Sciences Publication (C) Convriaht: All riahts reserved. 
24. L. van Hoesel, T. Nieberg, W. Wu Jian, and P. Havinga, "Prolonging the lifetime of wireless sensor networks by cross-layer interaction," IEEE wireless commu-nications, vol. 11, no. 6, pp. 78-86, 12 2004, doi:10.1109/MWC.2004.1368900 (see also 1536-1284).

25. R. Madan, S. Cui, S. Lall, and A. J. Goldsmith, "Model-ing and optimization of transmission schemes in energy-constrained wireless sensor networks," IEEE/ACM Trans-actions on Networking, vol. 15, no. 6, pp. 1359-1372, 2007.

26. T. Otsuka, T. Inamoto, Y. Torii, and T. Ito, "A high-speed sensor resources allocation method for distributed wsn," 10 2015, pp. 242-246.

27. I. E. L. Qachchach, A.-K. Yazbek, O. Habachi, J.-P. Can-ces, and V. Meghdadi, "New concatenated code schemes for data gathering in wsn's using rank metric codes," 2018 IEEE Wireless Communications and Networking Conference (WCNC), pp. 1-6, 2018.

28. K. Xie, L. Wang, X. Wang, G. Xie, and J. Wen, "Low cost and high accuracy data gathering in wsns with matrix completion," IEEE Transactions on Mobile Com-puting, vol. 17, no. 7, pp. 1595-1608, 2018.

29. S. Ghosh, S. Mondal, and U. Biswas, "Enhanced pegasis using ant colony optimization for data gathering in wsn," 2016 International Conference on Information Communi-cation and Embedded Systems (ICICES), pp. 1-6, 2016.

30. M. Abo-Zahhad, M. Farrag, and A. Ali, "Modeling and minimization of energy consumption in wireless sensor networks," in 2015 IEEE International Conference on Electronics, Circuits, and Systems (ICECS), 2015, pp. 697-700.

31. A. Krolo, B. Rzepka, and B. Bertsche, "Application of bayes statistics to reduce sample-size, considering a lifetime-ratio," in Annual Reliability and Maintainability Symposium. 2002 Proceedings (Cat. No.02CH37318), 2002, pp. 577-583.

32. J. Liu, X. Jiang, H. Nishiyama, and N. Kato, "Delivery ratio in two-hop relay manets with limited message lifetime and redundancy," in 2012 IEEE International Conference on Communications (ICC), 2012, pp. 5173- 5177.

\section{AUTHORS PROFILE}

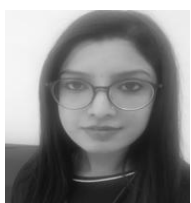

Sinduja M S received M.Tech. degree in Electronics and Communication Engineering from visvesvaraya technological university (VTU), India in 2016. She is currently pursuing Ph.D. with visvesvaraya technological university (VTU), India. Her research interest includes Wireless Sensor Networks, Route Discover Mechanism.

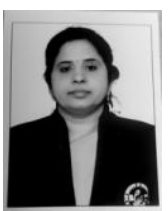

Dr. Rekha K. $\mathbf{R}$ received $\mathrm{PhD}$ in the area of Electronics and Communication and working as Professor in the department of ECE at SJB Institute of Technology, Bengaluru, India. Her re- search area mainly consists of network design, high speed optical transmission, and wireless sensors. She Published papers in National and International conference and Journals. And published totally 98 articles in various Journals and Conferences.

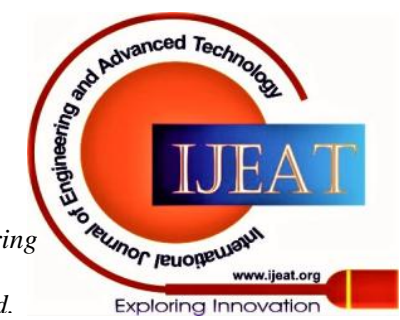

